\title{
Fuchs' Heterochromic Uveitis: A Reappraisal of the Clinical Spectrum
}

\author{
NICHOLAS P. JONES \\ Manchester
}

\begin{abstract}
Summary
The varying clinical appearance within a group of 103 patients with Fuchs' Heterochromic Uveitis (FHU) is discuissed. Bilateral cases accounted for $7.8 \%$ of patients. Cataract was found in $80.2 \%$ of cases after 8.8 years mean follow-up, and glaucoma affected $26.2 \%$ of patients. The most important diagnostic criteria are the typical pattern of iris atrophy and depigmentation, the characteristic intraocular inflammation, and complicated cataract. These are described in detail. The correct diagnosis of FHU is important because it affects future management and prognosis. The presence or absence of macroscopic heterochromia is not a helpful component of the ocular examination and should play little part in diagnosis. FHU does not necessarily present in its classical form, and may first resemble acute anterior uveitis or pars planitis. The syndrome may be the end stage of a number of different conditions.
\end{abstract}

\begin{abstract}
Although the combination of heterochromia and cataract was noted in 1843 by Lawrence, ${ }^{1}$ and later by others ${ }^{2,3}$ it was not until 1906 that Ernst Fuchs comprehensively described a group of 38 patients with signs of a form of heterochromia with inflammation and cataract, dubbed 'complicated heterochromia'. The syndrome which now bears his name has interested many authors in the succeeding 85 years and some series of considerable size have been published. ${ }^{5-8}$. The classical diagnostic criteria for the condition were reiterated in 1955 by Franceschetti ${ }^{5}$ and include the presence of stellate keratic precipitates, a characteristic pattern of iris atrophy, with or without nodules (and without posterior synechiae), vitreous opacification, cataract and possibly glaucoma. A comprehensive review of the literature was published in 1973. ${ }^{9,10}$

The critical examination of a large number of patients with Fuchs' Heterochromic Uvei-
\end{abstract}

tis (FHU) has placed into perspective some of the accepted criteria for diagnosis, has allowed further comment on some aspects of previous reports, and has brought to light some features not previously discussed in detail. The clinical spectrum of FHU is wider than has been previously defined, and the clinical course is more variable. Studies on a cohort of 103 patients with FHU are reported.

\section{Patients and Methods}

Commencing in 1987, all new and old patients seen at the Manchester Royal Eye Hospital with a definite or suggested diagnosis of FHU, were referred to and examined by the author. In all cases a detailed history was taken, including mode of, and reason for, presentation. General medical, neonatal, gestational and family histories were included. The clinical course of the disease process was 
noted, including a record of medical and surgical management.

A full clinical examination included appraisal of facial appearance, slit-lamp biomicroscopy including a critical examination of cornea, anterior chamber and iris, applanation, and dilatation for fundoscopy. Corneal endothelial specular microscopy and haematological investigations were performed in selected cases. Most patients have been examined on several occasions by the author. A total of 128 patients were referred with a definite or suggested diagnosis of FHU. Of these, 103 were considered to fulfil criteria for this diagnosis and were included in the survey.

\section{Results}

The patients' ages at presentation are shown graphically in Fig. 1. The range was 8 to 71 years, with a mean of 36.1 years. The major symptoms at presentation are shown in Table I. The follow-up since presentation has a range of six months to 59 years, with a mean of 8.8 years. Fifty patients $(48.5 \%)$ were male and $53(51.5 \%)$ female. Ninety-five patients $(92.2 \%)$ were Caucasian, three Asian and five Afro-caribbean or mixed race (total $7.8 \%$ non-Caucasian). The precise catchment population of this hospital cannot be defined, but the racial balance of these patients probably does not indicate any racial predilection for the disease.

The diagnosis of FHU was not always made immediately on presentation. The delay in diagnosis ranged from 0 to 57 years, with a mean of 3.4 years. Table II shows the diagnoses made on presentation.

The degree of heterochromia was graded $0-4$ by the author. Heterochromia was absent (Grade 0) in 10 patients, of whom eight $(7.8 \%)$ had bilateral disease and two had dark brown irides. Heterochromia Grade 1-4 was present in the remaining 93 patients $(90.3 \%)$ but was extremely subtle (Grade 1) in 42 cases, though usually still apparent with the naked eye. In a small number of patients, heterochromia could only be defined by comparison of anterior segment photographs.

Of those patients with heterochromia, 40 $(43.0 \%)$ were alreadyaware of this fact. All of these patients knew the time of onset of their heterochromia, and appeared to fall into two distinct groups; fifteen patients had congenital heterochromia and 25 had noticed a later onset. The age at which the heterochromia was first noticed, for the 25 patients in which it was not congenital, is shown in Fig. 2).

A previous ophthalmic history revealed that 16 patients $(15.5 \%)$ had had poor vision in the affected eye since childhood, but none had presented to an ophthalmologist at that stage. A lower best visual acuity on the affected side (in those with clear media and no macular disease) implied amblyopia. There was no history of strabismus in any of these patients. Only three patients had significant anisometropia, the affected eye being more myopic in all three cases. No patient had evidence of amblyopia in the eye without inflammation. Of these patients with amblyopia, nine were aware of their heterochromia, and in four cases it was definitely acquired, at ages 17, 21, 35 and 38 years, respectively. Two patients remembered a blunt anjury of some significance to the involved eye at some time previously. Nineteen patients complained of eye or brow ache before or since presentation.

Three patients were aware of a significant maternal illness during pregnancy. One mother had serum hepatitis, one had epileptic fits (the reason being unknown), and another required transfusion. Ninety-eight patients were delivered vaginally at term without incident. Five patients were delivered with forceps, of whom one was known to have sustained an ocular injury, and one claimed to have been injured. The only significant problem in the neonatal period involved one patient with a ventricular septal defect which required closure.

In no patient in this series was evidence found of a positive familial association for FHU or any other variety of heterochromia. On the contrary, discordance in a pair of monozygotic twins has been discovered. The implications of this on any genetic theory for FHU are discussed elsewhere. ${ }^{11}$

Enquiries into the general health of these patients revealed the following; three patients have peptic ulceration, two systemic hypertension, and one each have Addison's disease, hypernephroma, diabetes, epilepsy, pernicious anaemia, Parkinsonism, ischaemic heart disease, discoid lupus erythematosus, 


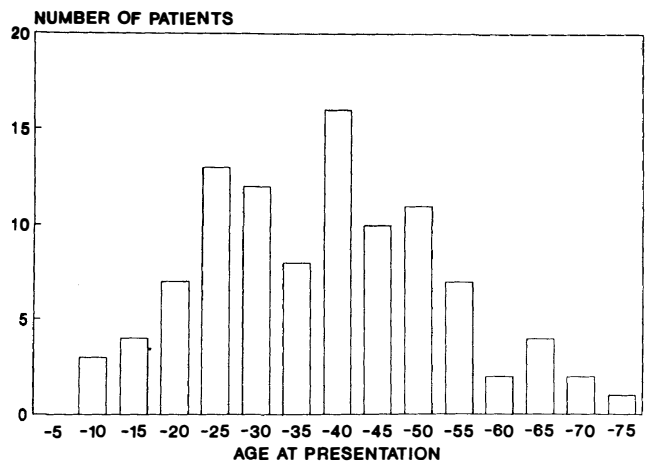

Fig. 1. The age range of patients with FHU at first presentation to an ophthalmologist.

previous tuberculosis, and Factor VII deficiency. One patient is a haemophilia carrier.

On examination, the right eye was affected in 50 cases $(48.5 \%)$ and the left in $45(43.6 \%)$, the remaining eight patients being bilateral. Head or facial appearance was notable in only two cases, both of whom were affected by the Parry-Romberg syndrome of hemifacial atrophy on the affected side. Ten patients had a partial ptosis on the affected side, of whom eight had undergone cataract surgery. No patient in this series had acceptable evidence of Horner's syndrome.

The cornea of the affected eye, examined with the slit-lamp biomicroscope, was abnormal in 17 eyes $(15.3 \%)$ of whom eight had previously undergone cataract surgery. Three corneas were partially or completely decompensated, two following cataract surgery. Eight corneas $(7.2 \%)$ exhibited a peripheral, circumferential haze of the deep stroma and endothelium. Two endothelia had numerous guttata. Three endothelia exhibited a striking abnormality consisting of multiple scalloped-out patches which were

Table I Major presenting symptoms in 103 patients with Fuchs's heterochromic uveitis. (NB some patients have more than one presenting symptom)

\begin{tabular}{lc}
\hline Symptom & $\begin{array}{c}\text { Number of } \\
\text { patients }\end{array}$ \\
\hline Decreased vision & 54 \\
Floaters & 34 \\
Discomfort & 12 \\
Heterochromia/uveitis noted by optician & 6 \\
$\quad$ or other doctor & 6 \\
Other & \\
\hline
\end{tabular}

acellular on specular microscopy, and one cornea showed linear marks in Descemet's membrane typical of previous trauma and consistent with a history of forceps damage during delivery.

Keratic precipitates (KP) were present in 93 eyes $(83.8 \%)$. The distribution of precipitates was variable, yet seemed to fit into three broad patterns (Fig. 3); generalised, affecting the whole posterior cornea (57 eyes); central (16 eyes); and inferior (20 eyes), often diffusely so but occasionally in a triangular distribution more commonly associated with acute anterior uveitis. The morphology of KP was variable, but many eyes $(45,40.5 \%)$ showed precipitates previously described as typical of FHU, ${ }^{5,12}$ that is, stellate with fibrillary extensions, and tiny interspersed fibrils. A diagrammatic example of a typical area of such keratic precipitates is shown in Fig. 4. Nineteen eyes $(17.1 \%)$ had KP which are described as 'glassy', being medium-sized, globular, transparent and non-pigmented. Old pigmented KP were seen in five eyes.

There was some correlation between morphology of KP and their distribution. Typically stellate KP were centrally or generally distributed ( 38 of 45 eyes, $84.4 \%$ ). Only seven of 20 eyes $(35 \%)$ with inferiorly-distributed KP showed stellate characteristics.

Anterior chamber activity was recorded traditionally on a scale of 0 to +++ . Fiftynine eyes $(53.1 \%)$ had no cellular activity and 49 eyes $(44.1 \%)$ had few cells only $(+)$. However, two eyes had ++ cells and one eye, +++ . Anterior chamber flare was an uncommon finding. Sixteen eyes $(14.4 \%)$ had typical signs of FHU including iris atrophy, heterochromia and cataract, yet had lost any evidence of intraocular inflammation (no active

Table II The initial diagnosis made in the 103 patients eventually diagnosed as Fuchs's heterochromic uveitis

\begin{tabular}{lrr}
\hline & $\begin{array}{c}\text { Number of } \\
\text { patients }\end{array}$ & $\%$ \\
\hline Fuchs's heterochromic uveitis & 65 & 63.1 \\
Acute anterior uveitis & 20 & 19.4 \\
Pars planitis & 8 & 7.8 \\
Phakolytic glaucoma & 1 & 1.0 \\
No diagnosis made & 9 & 8.7 \\
\hline Total & 103 & 100.0 \\
\hline
\end{tabular}




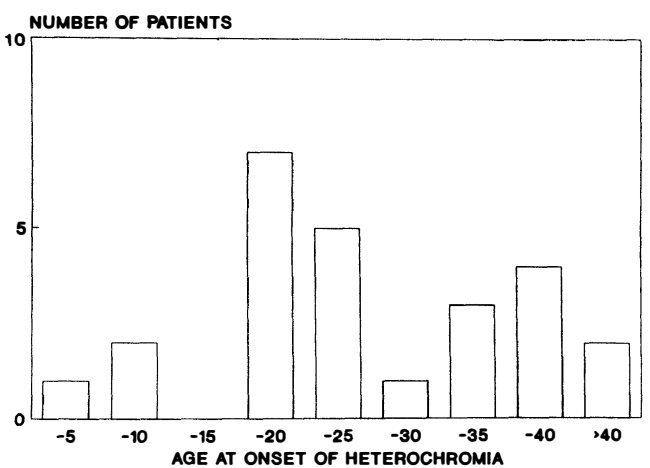

Fig. 2. The age at which heterochromia was first noticed by those patients in whom it was acquired.

KPs, no cellular activity in anterior chamber or vitreous, no iris nodules). In all 16 cases the eye had previously undergone cataract surgery.

Examination of the iris included a separate assessment of the degree of atrophy of anterior border layer, stroma and pigment epithelium on a scale of 0 (normal) to three (very severe), and the presence or absence of nodules, vessels or synechiae was noted. The findings in relation to iris atrophy are shown in Table III. The vast majority of patients had complete loss of the anterior border layer pigment. The pattern of pigment epithelial atrophy was extremely variable but a particular predilection was noted for the area adjacent to the pupil (affected alone in 16 cases, $14.4 \%$ ) and the iris periphery (affected alone in 10 cases, $9.0 \%$ ). The remaining 35 patients with pigment epithelial atrophy showed scattered transillumination defects. No patient had an atrophic sector. The pigment ruff appeared particularly vulnerable and was patchy or absent in 59 eyes $(53.1 \%)$.

Iris nodules were a frequent feature in these patients. Fifteen patients had active Koeppe nodules, four had Busacca nodules covering

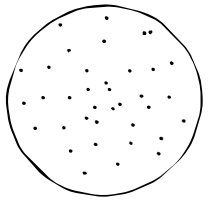

WIDESPREAD

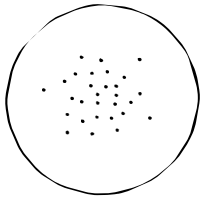

CENTRAL

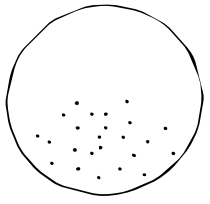

INFERIOR
Fig. 3. The distribution of $K P$ in this series: Three broad categories. the entire iris surface (but always more numerous in the pupillary area, Fig. 5) and one patient had numerous refractile Russell bodies. Cicatricial posterior synechiae were seen in four patients, all of whom had previously had cataract surgery. Although no transient synechiae were seen, in five patients who were phakic, radial stripes of iris epithelial pigment were seen on the anterior lens surface, indicating previous adhesion (Fig. 6).

Iris neovascularisation was an uncommon finding but was definitely observed in six eyes and is discussed elsewhere. ${ }^{17}$ Iris haemorrhage following paracentesis or during cataract surgery has previously been discussed. ${ }^{13,14}$ In this survey, filiform haemorrhage following applanation tonometry was observed in two eyes on one occasion each.

The crystalline lens was completely clear in 22 eyes (19.8\%). The remaining 89 eyes had a degree of cataract (32 eyes, $28.8 \%$ ) or had undergone cataract surgery (57 eyes, $51.3 \%$ ). Of these latter, posterior chamber intraocular lenses (IOLs) were implanted in 27 cases, iris supported IOLs in six cases, and the rest underwent cataract extraction without IOL implantation. The results of modern extracapsular cataract surgery in FHU have now been described, ${ }^{14-16}$ including 30 of the above 57 eyes. ${ }^{14}$ The mean duration of disease since diagnosis was 10.4 years for those with cataract and 6.0 years for those with clear lenses. A small number of patients in this series had

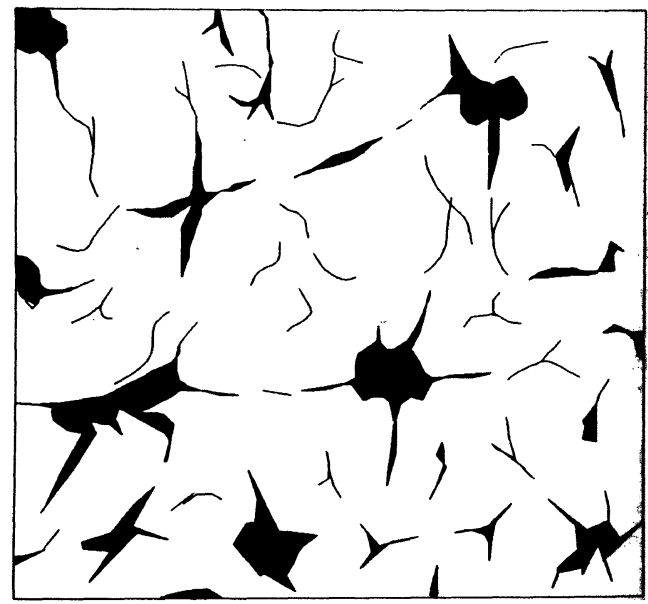

Fig. 4. A drawing of a small area of typical $K P$ in $F H U$, being stellate or dendritic with fine interspersea fibrils. 
Table III Degree of iris atrophy, on a scale of 0-3, for each iris layer (number of patients in each category)

\begin{tabular}{lrrrr}
\hline & \multicolumn{4}{c}{$\begin{array}{c}\text { Degree of } \\
\text { iris atrophy }\end{array}$} \\
\cline { 2 - 5 } Iris Layer & 0 & 1 & 2 & 3 \\
\hline Anterior border layer & 5 & 17 & 16 & 73 \\
Stroma & 12 & 47 & 43 & 9 \\
Pigment epithelium & 50 & 32 & 23 & 6 \\
\hline
\end{tabular}

stellate precipitates on the back of the crystalline lens, always inferiorly, yet none were seen on the anterior surface.

At some stage in the disease process, 27 patients $(26.2 \%)$ were treated for glaucoma. The aetiology, management and outcome of the glaucoma in these patients are reported elsewhere. $^{17}$

Vitreous opacification of some degree was present in 74 eyes $(66.6 \%)$, consisting of cellular activity, deposition of precipitates on vitreous architecture (some of these clearly stellate), and veil formation. Three of these patients had complete opacification, necess-

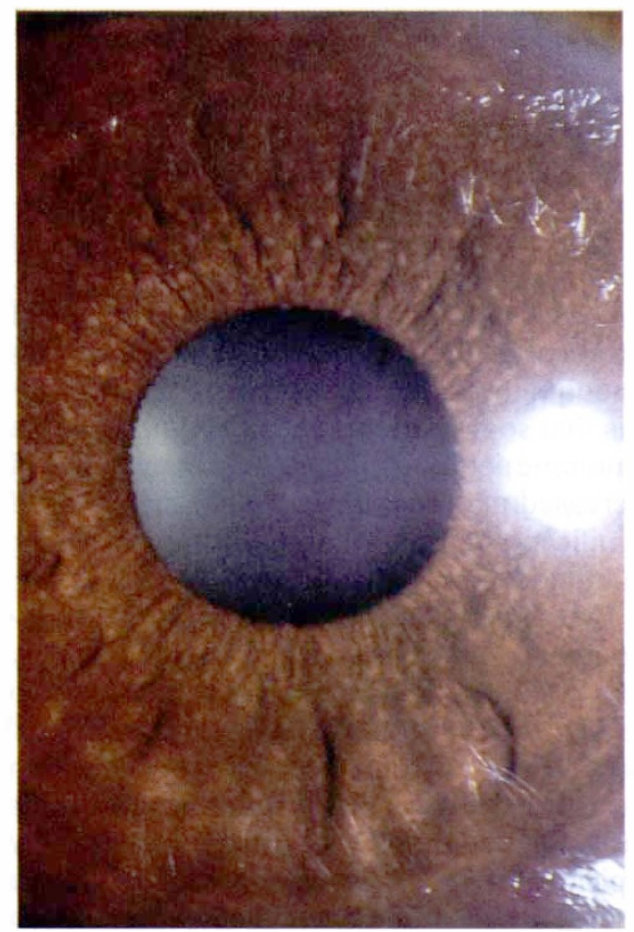

itating vitrectomy. Of the remaining 47 eyes, 15 could not be assessed owing to cataract formation. The remaining 22 eyes $(19.8 \%)$ had completely clear vitreous humour.

Examination of the fundus was performed wherever possible, but there was an inadequate view in 10 eyes. Choroidal scars resembling old toxoplasmosis were present in eight eyes $(7.2 \%)$, as reported in other series..$^{18,19}$

\section{Discussion}

Some of the above information merely reiterates and reinforces previous reports on FHU. There are however certain features worthy of separate discussion:

\section{A. The History}

This survey draws attention to various useful features of the history which may suggest FHU. In this series visual loss, usually due to cataract formation, was the commonest reason for presentation, but a complaint of floaters is also frequent and in this age group should alert one to the possibility of FHU. Ocular or periocular aching, although pre-

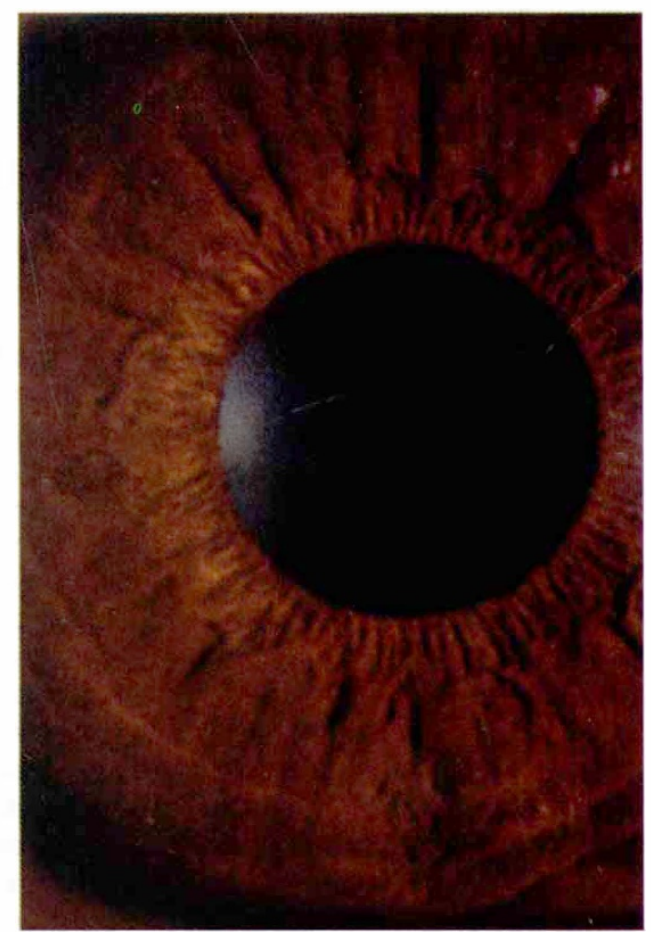

Fig. 5. FHU affecting the right eye with mild heterochromia. Both Koeppe (pupil margin) and Busacca (iris surface) nodules are present. 

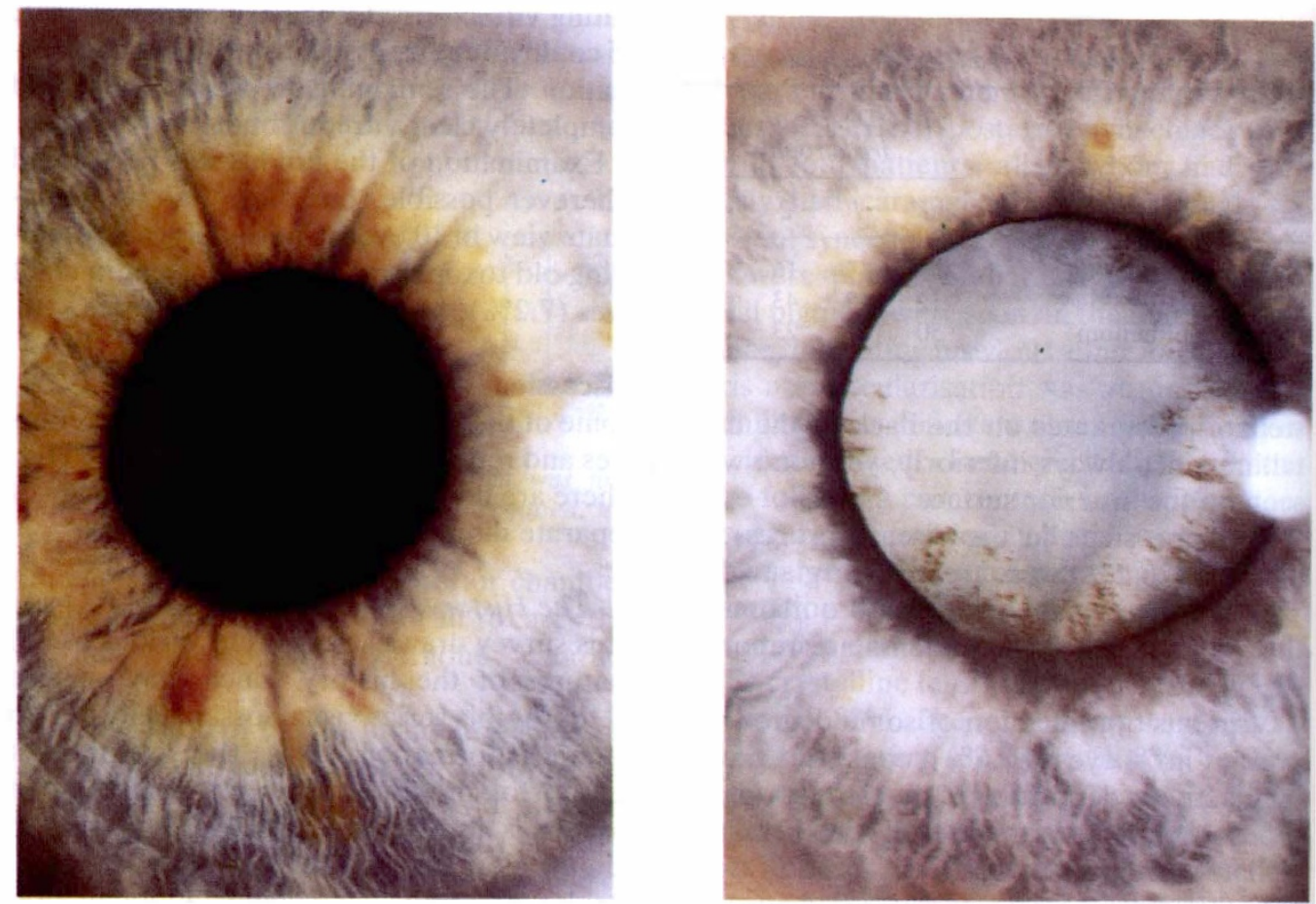

Fig. 6. FHU affecting the left eye. Complete absence of anterior border layer pigment, and radial stripes of iris epithelial pigment de posited on the anterior lens surface, probably indicating previous sites of adhesion by 'sticky' Koeppe nodules.

viously mentioned in connection with $\mathrm{FHU},{ }^{7,8}$ was found to be common in this series, affecting 19 patients $(18.4 \%)$, but was not associated with an identifiable cause, such as ciliary spasm or raised intraocular pressure, in contrast to Liesegang's patients. ${ }^{7}$

Sixteen eyes with FHU had poor visual acuity of long standing, which could not be explained fully by their current disease process. No patient had evidence of strabismic amblyopia, and only one patient had gross anisometropia, the axial length of the affected eye being $3.5 \mathrm{~mm}$ greater than its fellow. Fuchs' heterochromic uveitis can commence in childhood. In the absence of another cause of amblyopia, it is possible that intraocular inflammation in childhood, with significant vitreous opacification, may lead to a degree of stimulus deprivation. This possibility seems unlikely however, and at present the cause of this high occurrence of amblyopia is unknown. It has not previously been mentioned in reports of FHU and should suggest FHU rather than another form of uveitis.
A traumatic aetiology for FHU has been suggested in the past. Several patients in this series reported significant trauma, but the significance of this is dubious. One patient had evidence of forceps injury during parturition. No other patient had acceptable evidence of intraocular damage from injury. There was inadequate evidence to support the hypothesis that some patients with FHU may have a traumatic aetiology. There was no evidence of increased maternal illness in pregnancy or of significant acquired illness during infancy or childhood.

Two patients in this series have the ParryRomberg syndrome of hemifacial atrophy. This condition has previously been linked to FHU, ${ }^{20,21}$ yet Loewenfeld and Thompson ${ }^{20}$ have disputed the connection. The two patients in this series have mild chronic uveitis with stellate keratic precipitates, iris atrophy typical of FHU, and other features of the disease. The argument ${ }^{10}$ that patients with hemifacial atrophy have heterochromic uveitis of a different aetiology to patients with 


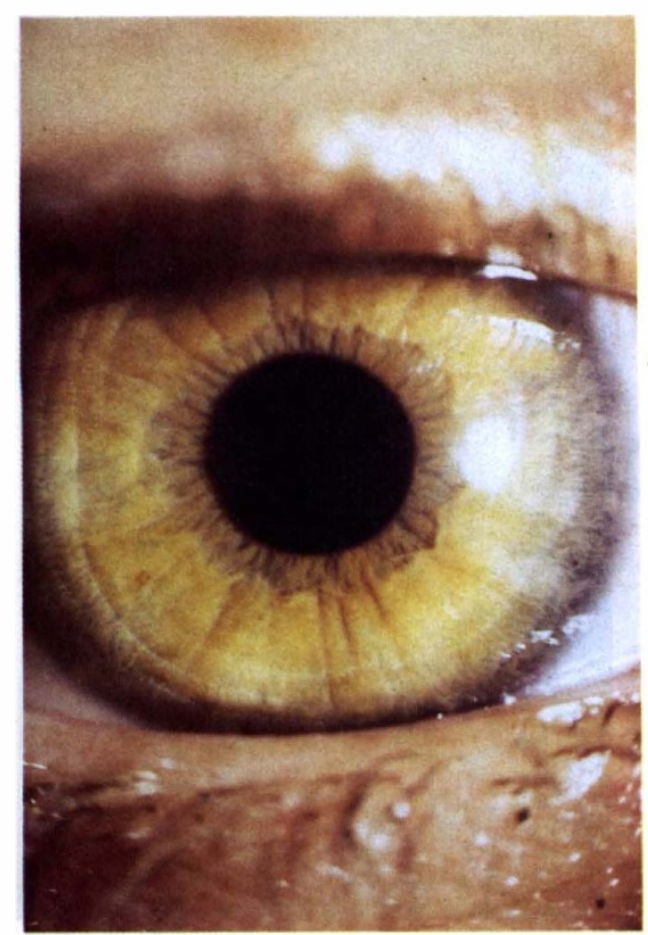

Fig. 7. Profound heterochromia due to left-sided FHU. The heterochromia is entirely due to loss of anterior border layer pigment.

Fuchs' syndrome is largely semantic. A single aetiology for all patients with FHU is an assumption which may prove to be incorrect. The above, reiterated association with the Parry-Romberg syndrome may provide one example of a different aetiology for FHU.

\section{B. The Iris}

The normal iris has an immense variability in structure and pigmentation, ${ }^{22}$ yet certain anatomical features allow the identification of changes common to all patients with FHU. It is convenient to consider three separate layers in this clinical context.

\section{a. The Anterior Border Layer}

The anterior border layer (ABL) is seen as an anterior stromal condensation, covering a variable proportion of the iris, often fenestrated, most dense about the collarette, and of extremely variable pigmentation. It is most easily visible when dense, and when stromal pigmentation is light. It is commonly seen as an orange blush when the stroma is blue, but is usually indistinguishable when the stroma is

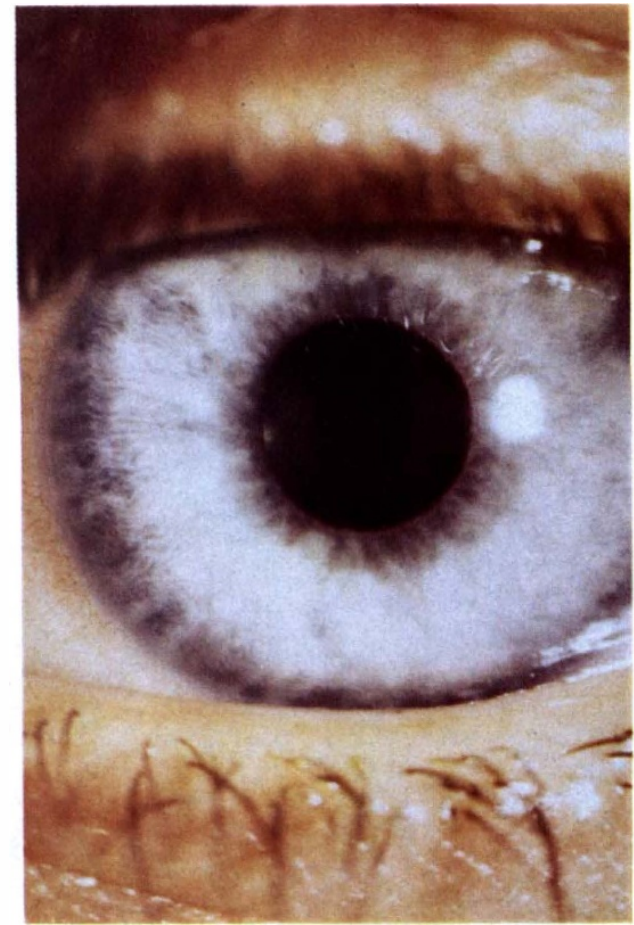

dark brown. It appears to be characteristic of FHU that atrophy of the ABL occurs early in the course of the disease. The vast majority of the above patients had severe ABL atrophy with complete depigmentation (Fig. 7). In some patients the depigmented $\mathrm{ABL}$ is revealed as whitish areas on the stromal surface (Fig. 8).

It is the depigmentation of this layer above all others, which is responsible for heterochromia in FHU.

\section{b. The Stroma}

Visibility of stromal architecture is dependent on the degree of stromal pigmentation. The denser the pigmentation, the less well defined the structure. At one extreme, a light blue stroma will have an easily visible radial fibrillary structure, visible (normal) radial vessels, a visible sphincter pupillae, and in some cases direct visibility of the pigment epithelium through absolute stromal defects (crypts). At the other extreme, a dark brown iris is choked by pigment-containing cells to the extent that a featureless velvety texture is apparent. 

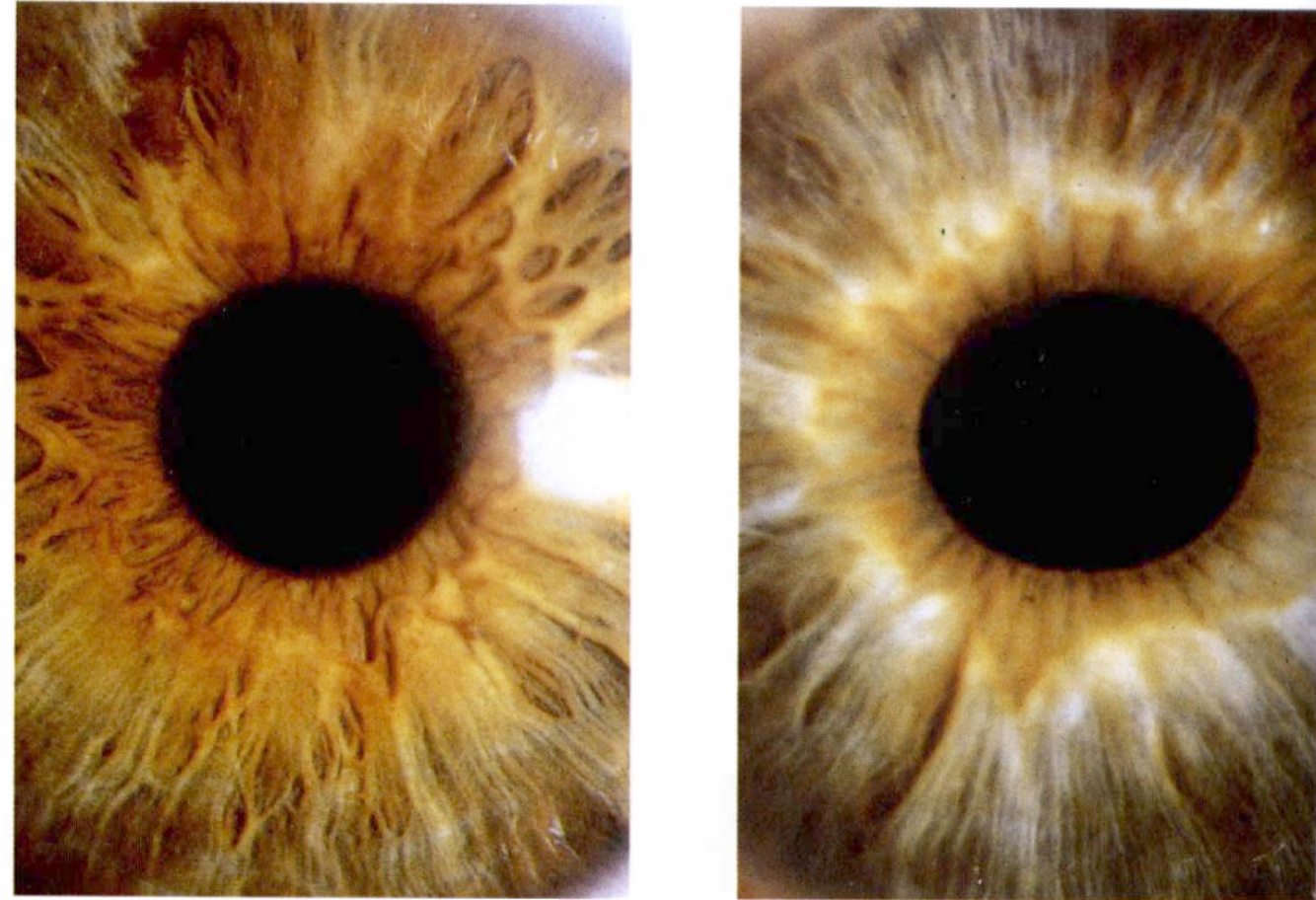

Fig. 8. FHU, in an early stage, affecting the left eye. The anterior border layer is becoming depigmented, being revealed as a whitish layer, most prominent around the collarette.

This variability explains two interesting phenomena in FHU. Firstly, progressive atrophy of a light blue stroma will increasingly reveal the pigment epithelium and cause an apparent deepening of the blue colour ${ }^{23}$ (socalled inverse heterochromia). Secondly, a profound degree of stromal atrophy is necessary in dark brown irides before macroscopic heterochromia becomes evident. The deep excavation of stroma is usually evident on the biomicroscope long before heterochromia is achieved (Fig. 9). Heterochromia is undoubtedly more difficult to see in those with dark brown, ${ }^{8,24}$ and light blue irides.

In general, FHU causes stromal atrophy of a type which highlights rather than hides its architecture; pigment and volume are lost, vessels become visible, the sphincter pupillae becomes visible even in dark brown eyes (Fig. 9) and crypts appear with exposed pigment epithelium at their base. Comparison with the unaffected eye is essential to appreciate these changes with the slit lamp, and simultaneous comparison of $35 \mathrm{~mm}$ colour transparencies of the irides often shows a degree of asymmetry which was not appreciated by macroscopic or slit-lamp examination.

In this sample of patients, stromal atrophy was common but less so than atrophy of the ABL (Table III). Stromal atrophy is more difficult to perceive than $\mathrm{ABL}$ atrophy and therefore is seen clinically at a later stage in the course of FHU.

\section{The Pigment Epithelium}

Abnormalities of the pigment epithelium in this series were seen as transillumination defects, and as fallout of the pupillary pig. ment ruff. The predilection of the former for the pupil area is an important sign in FHU, though by no means pathognomonic. In some severe cases there was virtually complete transillumination of the pigment epithelium, yet these irides were notable for their complete lack of posterior synechiae. Transillumination of the pigment epithelium was an inconstant finding in this series and tended to occur after atrophy of the other layers.

FHU causes atrophy and depigmentation 

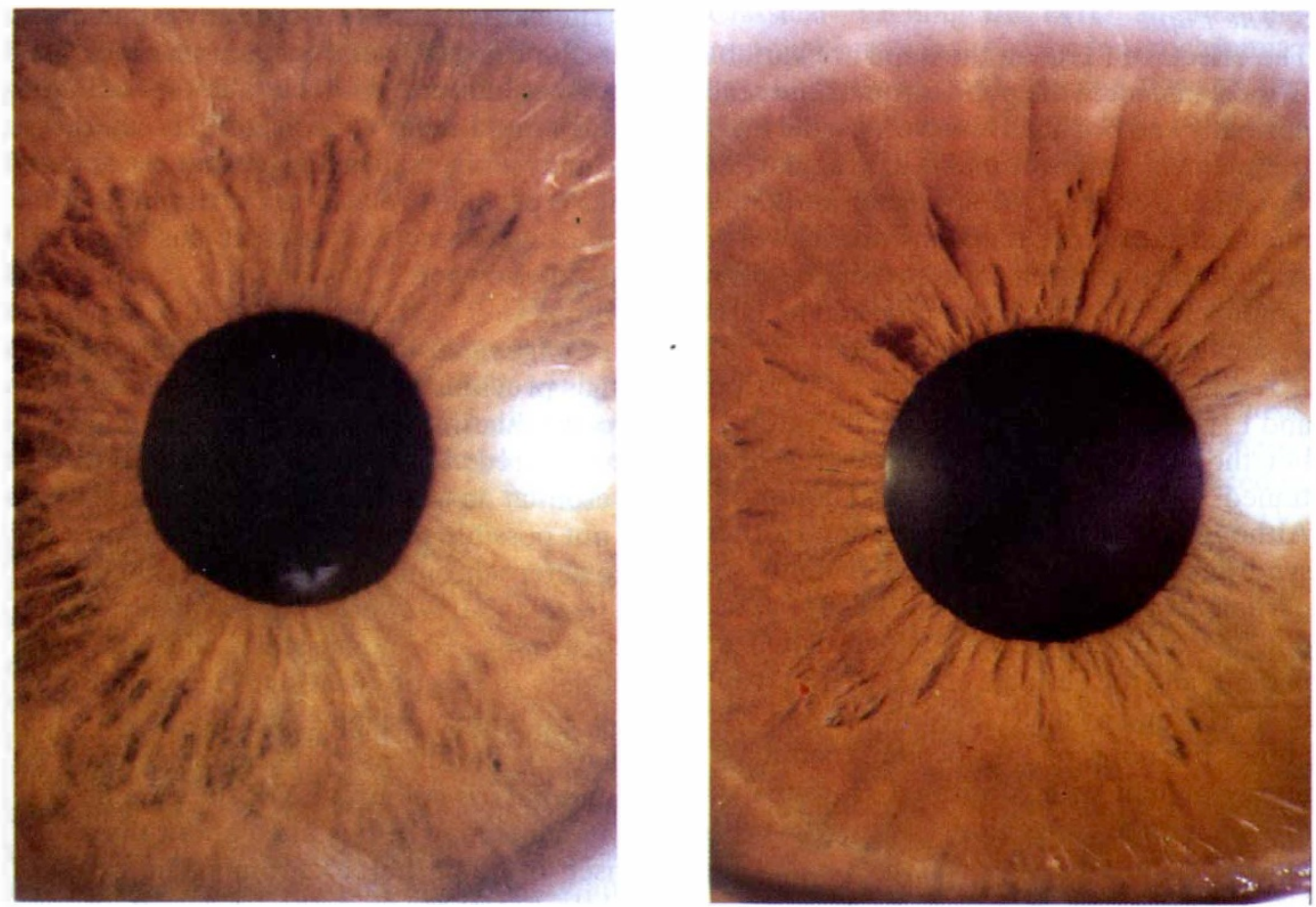

Fig. 9. Late-stage FHU affecting the right eye. There is severe stromal atrophy with increased visibility of the radial structure, and the sphincter pupillae is now visible. The heterochromia is very subtle.

of all iris layers, yet clinically the layers are affected in the order: ABL; Stroma; Pigment epithelium. This does not imply any temporal sequence but merely the ease with which atrophy and depigmentation become visible with the biomicroscope. Sectorial iris atrophy was not found in this series and this finding should strongly suggest a different diagnosis.

\section{Vessels, nodules and synechiae}

Abnormal vasculature within the iris stroma and in the anterior chamber angle has been described in association with $\mathrm{FHU}^{25-28}$ yet except where gross rubeosis is concerned, such findings are open to variable interpretation. Henkind ${ }^{29}$ points out that 'abnormal' angle vessels, described previously in FHU, may be erroneously described. The normal iris vasculature will naturally become more easily visible following atrophy of $\mathrm{ABL}$ and stroma and this may explain the finding of unduly prominent iris blood vessels by some. Frank rubeosis has been noted in the later stages of FHU in this series, in association with glaucoma following intracapsular catar- act surgery. This survey has not revealed widespread vascular abnormalities in FHU, but iris angiography has clearly demonstrated subclinical changes in the past. ${ }^{27,28,30}$

The presence of iris nodules has previously been noted to be an occasional feature of FHU, ${ }^{5}$ but is certainly not rare, as stated by O'Connor, ${ }^{31}$ affecting 18 eyes $(16.2 \%)$ in this series. These nodules were inevitably small, virtually transparent, and numerous. They did not appear to be associated with more severe inflammation.

Previous reports rightly emphasise the absence of posterior synechiae in $\mathrm{FHU}^{5,7,31}$ but the condition does not protect from synechia formation after cataract surgery. Uveitis following cataract surgery was the cause of cicatricial posterior synechiae in four eyes. Transient synechiae have also been reported and are thought to represent adhesions of Koeppe nodules to the anterior lens capsule. In this series no synechiae were seen in association with Koeppe nodules but radial stripes of pigment deposition on the lens capsule probably represent previous sites of adhesion. 


\section{The Pupil}

The effects of FHU on the pupil are variable. In most instances the pupil is round and reacts normally and briskly. However, partial loss of the pigment ruff will make it irregular, and variable atrophy of the sphincter and dilator pupillae can render reactions untrustworthy. Anisokoria was seen in many of the above patients but the affected pupil could be larger or smaller than the unaffected side.

A connection between Horner's syndrome and FHU has been noted in the literature ${ }^{32-34}$ but the association has been severely questioned by Loewenfeld and Thompson. ${ }^{10}$ Although the question of coexisting Horner's syndrome was raised in those patients with partial ptosis in this series, the diagnosis could not be confirmed pharmacologically in any patient, and the variable effects of iris atrophy in FHU make such tests unreliable. The author has not seen intraocular inflammation in any patient with confirmed Horner's syndrome, either congenital (with heterochromia) or acquired.

\section{c. Cornea, lens and media}

This series exhibits a significant number of patients with corneal problems, a feature not discussed at length previously. Alanko et al. ${ }^{35}$ showed morphological abnormalities of corneal endothelium in patients with FHU, and peripheral corneal oedema after cataract surgery has been noted in patients with $\mathrm{FHU}^{7}$ and other forms of uveitis. ${ }^{36}$ In this series, eight eyes had an abnormality of the deep periphery of the entire corneal circumference, seen as a semiopaque sheet on the slitlamp, and one patient had peripheral stromal thickening and epithelial oedema overlying the inferior $150^{\circ}$ of this. No gonioscopic abnormality was seen in association with this and the aetiology remains unclear.

The stellate nature of typical keratic precipitates in FHU has been extensively discussed. $^{5}$ In this series, a few patients in addition also showed stellate deposits on the posterior lens surface and within the vitreous cavity. Vitreous activity is usual in FHU, but in three patients in this survey, relatively sudden and dense vitreous opacification occurred in the absence of anterior segment inflammation, requiring vitrectomy. Such a complication has not previously been described.
The cataract in FHU typically commences as a posterior subcapsular cataract and becomes mature with variable speed. This study would seem to indicate that cataract is virtually universal in FHU, and its appearance is probably related to the duration of the disease. Surgery for cataract has been discussed elsewhere. ${ }^{14-16}$

\section{Congenital or acquired heterochromia}

It has previously been noticed ${ }^{5,7}$ that the heterochromia in FHU can be either congenital or acquired. Since patients with FHU often present at an advanced state of the disease, it is not possible to confirm the age of onset of the heterochromia. The very fact that only 40 patients $(43.0 \%)$ were aware of their own heterochromia demonstrates the frequent subtlety of this physical sign. Heterochromia will of course become visible at different stages of the disease process, depending on the initial iris colouration of the patient.

Patients may be mistaken about the age of onset of their heterochromia. In cases of subtle heterochromia this is certainly possible. However, some patients in this series have very obvious heterochromia of the order of severity shown in Fig. 7. All nine patients with heterochromia Grades 3 or 4 were aware of it. Of these, four maintained that it was congenital, and five had acquired it, at ages 4 , $18,20,24$ and 40 years respectively. We have to assume that these patients are correct in their estimation of the age of onset of heterochromia. The heterochromia in FHU may therefore be congenital or acquired.

Is FHU present, either constantly or intermittently, from birth in those patients with congenital heterochromia? If this is so then we would expect those with congenital heterochromia to present significantly earlier than those with acquired heterochromia, as the two forms appear clinically similar in every other parameter. In fact this is not the case. The fifteen patients with congenital heterochromia presented at a mean age of 41.4 years, and the 25 with acquired heterochromia, 33.7 years. It seems unlikely that FHU is present from birth in those with congenital heterochromia.

The remaining possibility is that those with congenital heterochromia have no intraocular 
inflammation at first (i.e. they have 'simple' heterochromia) but later develop FHU. Congenital heterochromia may prove to be one of a number of factors which predispose to the future development of FHU.

Simple heterochromia is thought to be familial, ${ }^{37.38}$ and simple heteróchromia and FHU have occasionally been reported together in the same family. ${ }^{7.39 .40}$ The discovery of discordance in monozygotic twins ${ }^{11}$ disproves the possibility of Mendelian inheritance, but the above hypothesis may explain the infrequent concurrence of simple heterochromia and FHU within the same family.

\section{E. The concept of burnt-out FHU}

It has on occasion been noted that eyes with FHU may lose. all signs of active inflammation, ${ }^{5.23}$ and this has been related to the removal of the lens. ${ }^{5}$ Signs of inflammation may reappear years after surgery. In this study 16 patients had lost all signs of inflammation and all of them had previously had cataract surgery. It is tempting to suggest a causal relationship between a lens abnormality and FHU but there is as yet inadequate evidence for this.

\section{Conclusion}

Previous authors on the subject of FHU comment on the frequent 'misdiagnosis' of the condition, as do many enthusiasts for their own subject. In this survey, 38 patients either had no diagnosis, or some other diagnosis made at the time of presentation, the label of FHU being applied later. There is a temptation to ascribe this to inadequate appraisal, yet the 'misdiagnoses' seem to follow a certain pattern (Table II) and there is another possible explanation which should be considered.

In several of these patients, in whom the diagnosis of FHU was beyond reasonable doubt, previous episodes of 'different' uveitis had been reliably recorded, such as a pars planitis with vitreous snowballs, or an acute iritis requiring intensive topical steroid treatment. In three patients the contralateral eye has been affected by acute fibrinous iritis, each on one occasion. It is tempting to suggest that FHU may represent a common end-stage of a variety of uveitides, and that there may be a period of a different clinical appearance before typical FHU becomes obvious.
Classical teaching on FHU stresses the paramount importance of the heterochromia. This assumption does an injustice to the disease and will undoubtedly lead to underdiagnosis. There is of course usually no heterochromia if the disease is bilateral (and it is bilateral in a significant minority of cases ${ }^{4,5,12}$ ). In any case the heterochromia is often very subtle. Critical biomicroscopy will reveal typical features of FHU and an appreciation of macroscopic heterochromia should only be used as confirmation of this. In the diagnosis of FHU in the above series, several 'hard' signs have been identified. They are placed in order of frequency of occurrence in Table IV, together with other signs of importance.

The differential diagnosis of FHU will include other mild forms of uveitis (apparently without heterochromia), lens-induced glaucoma and uveitis, pars planitis, and the Posner-Schlossmann syndrome, which frequently causes heterochromia. ${ }^{41}$ In some cases the diagnosis is extremely difficult to make. However, heterochromia without inflammation, such as 'simple' heterochromia, sympathetic heterochromia, and heterochromia in association with other conditions such as Duane's syndrome and Waardenburg's syndrome, should not provide a problem. Hyperchromic causes of heterochromia such as ocular melanosis, iris naevus syndrome and xanthochromia have typical features which should exclude them from the differential diagnosis.

Table IV Frequency of occurrence of some important symptoms or physical signs in this survey of 103 patients with Fuchs' heterochromic uveitis

\begin{tabular}{lcc}
\hline & \multicolumn{2}{c}{ Percentage affected } \\
\cline { 2 - 3 } Sign & Patients & Eyes \\
\hline Absence of posterior synechiae & & \\
$\quad$ (before intraocular surgery) & & 100.0 \\
Few AC cells (0 or 1+) & & 97.2 \\
ABL depigmentation & & 95.5 \\
Unilaterality & 92.2 & \\
Heterochromia & 90.3 & \\
Iris stromal atrophy & & 89.2 \\
Keratic precipitates & & 83.8 \\
Cataract & & 80.2 \\
Vitreous opacities & 66.6 \\
Glaucoma & & \\
Periocular aching & & \\
Iris nodules & & 16.2 \\
Amblyopia & 18.4 & \\
\hline
\end{tabular}


The diagnosis of FHU at present is entirely a clinical one, though the recent finding of frequent immunological abnormalities in these patients, compared with other uveitides, encourages us to anticipate a diagnostic test in the near future..$^{42-45}$ In the meantime, accurate clinical examination is the mainstay of diagnosis. Upon it rests the future management of the patient, and the prognosis, both of which may differ substantially from other forms of uveitis.

I am grateful to all medical staff at Manchester Royal Eye Hospital for their help in providing patients for the study. I am indebted to the staff of the Department of Retinal Photography, MREH, for their help with the production of illustrations.

Key Words: Fuchs' Heterochromic Uveitis, Heterochromia, Heterochromic cyclitis.

\section{References}

${ }^{1}$ Lawrence W: Changes in colour in the iris. In Hays $\mathrm{J}$ (Ed.) A Treatise on diseases of the eye. Lea \& Blanchard, Philadelphia 1843: 411-416.

${ }^{2}$ Bistis J: Heterochromie und Kataractbildung. $\mathrm{Zbl}$ Prakt Augenheilk 1898, 22: 136-7.

${ }^{3}$ Weill G: Ueber H heterophthalmus. Z Augenheilk 1904, 11: 165-76.

${ }^{4}$ Fuchs E: Ueber Komplicationen der Heterochromie. Z Augenheilk 1906, 15: 191-212.

${ }^{5}$ Franceschetti A: Heterochromic cyclitis (Fuchs' Syndrome) Am J Ophthalmol 1955, 39: 50-8.

${ }^{6}$ Huber A: Das Glaukom bei komplizierter Heterochromie Fuchs. Ophthalmologica 1961, 141: 122-35.

${ }^{7}$ Liesegang TJ: Clinical features and prognosis in Fuchs' uveitis syndrome. Arch Ophthalmol 1982, 100: $1622-6$.

${ }^{8}$ Tabbut BR, Tessler HH, Williams D: Fuchs' heterochromic iridocyclitis in blacks. Arch Ophthalmol 1988, 106: 1688-90.

${ }^{9}$ Loewenfeld IE and Thompson S: Fuchs's heterochromic cyclitis. A critical review of the literature I. Clinical characteristics of the syndrome. Surv Ophthalmol 1973, 17: 394-457.

${ }^{10}$ Loewenfeld IE and Thompson S: Fuchs's heterochromic cyclitis. A critical review of the literature II. Etiology and mechanisms. Surv Ophthalmol 1973, 18: 2-61.

${ }^{11}$ Jones NP and Read AP: Is there a genetic basis for Fuchs's heterochromic uveitis?: Discordance in monozygotic twins. Br J Ophthalmol 1991, (In Press).

${ }^{12}$ Kimura SJ, Hogan MJ, Thygeson P: Fuchs's syndrome of heterochromic cyclitis. Arch Ophthalmol 1955, 54: 179-86.

${ }^{13}$ Amsler M: New clinical aspects of the vegetative eye. Trans Ophthalmol Soc UK 1948, 68: 45-74.

${ }^{14}$ Jones NP: Extracapsular cataract surgery with and without intraocular lens implantation in Fuchs's heterochromic uveitis. Eye 1990, 4: 145-50.
${ }^{15}$ Gee SS and Tabbara KF: Extracapsular cataract extraction in Fuchs's heterochromic iridocyclitis. Am J Ophthalmol 1989, 108: 310-14.

${ }^{16}$ Jakeman CM, Jordan K, Keast-Butler J, Perry S: Cataract surgery with intraocular lens implantation in Fuchs's heterochromic cyclitis. Eye 1990, 4: 543-7.

${ }^{17}$ Jones NP: Glaucoma in Fuchs's heterochromic uveitis: Aetiology, management and outcome. Eye 1991, 5: 662-7.

${ }^{18}$ De Abreu MT, Belfort R, Hirata PS: Fuchs's heterochromic cyclitis and ocular toxoplasmosis. Am J Ophthalmol 1982, 93: 739-44.

${ }^{19}$ Arffa RC and Schlaegel TF: Chorioretinal scars in Fuchs' heterochromic iridocyclitis. Arch Ophthalmol 1984, 102: 1153-5.

${ }^{20}$ Perkins ES: Heterochromic uveitis. Trans Ophthalmol Soc UK 1961, 81: 53-66.

${ }^{21}$ Sugar HS and Banks TL: Fuchs's heterochromic cyclitis associated with facial hemiatrophy (scleroderma en coup de sabre). Am J Ophthalmol 1964, 57: 627-32.

${ }^{22}$ Fine BS and Yanoff $M$ : The uveal tract. In: Ocular Histology (2nd Ed.) Harper \& Row, New York 1979.

${ }^{23}$ Sugar HS: Heterochromia iridis with special consideration of its relation to cyclitic disease. $A m \mathrm{~J}$ Ophthalmol 1965, 60: 1-18.

${ }^{24}$ Jain IS, Gupta A, Gangwar DN, Dhir SP: Fuchs' heterochromic cyclitis: some observations on clinical picture and on cataract surgery. Ann Oph: thalmol 1983, 15: 640-2.

${ }^{25}$ Lerman $\mathrm{S}$ and Levy C: Heterochromic iritis and secondary neovascular glaucoma. Am J Ophthal. mol 1964, 57: 479-81.

${ }^{26}$ Perry HD, Yanoff M, Scheie HG: Rubeosis in Fuchs's heterochromic iridocyclitis. Arch ophthalmol 1975, 93: 337-9.

${ }^{27}$ Saari M, Vuorre I, Nieminen H: Fuchs's heterochromic cyclitis: a simultaneous bilateral fluorescein angiographic study of the iris. $\mathrm{Br} J$ Ophthalmol 1978, 62: 715-21

${ }^{28}$ Berger BB, Tessler HH, Kottow MH: Anterior seg ment ischaemia in Fuchs' heterochromic cyclitis: Arch ophthalmol 1980, 98: 499-501.

${ }^{29}$ Henkind P: Angle vessels in normal eyes. A gonioscopic evaluation and anatomic correlation. $\mathrm{Br} J$ Ophthalmol 1964, 48: 551-7.

${ }^{30}$ Pivetti Pezzi P, Bozzoni F, De Liso P, Catarinelli G, Nazziocone M: Iris fluorescein angiography in Fuchs's heterochromic iridocyclitis. Boll Oculist 1984, 63: 879-86.

${ }^{31}$ O'Connor GR. Heterochromic iridocyclitis. Trans Ophthalmol Soc UK 1985: 104: 219-31.

${ }^{32}$ Strieff EB. Considerations pathogenetiques surl'he, terochromie de Fuchs. Bull Mem Soc Fr Ophthal mol 1950; 63; 223.

${ }^{33}$ Makley TA and Abbott K. Neurogenic heterochro mia. Report of an interesting case. Am J Ophthat mol 1965, 59: 927-8.

${ }^{34}$ Regenbogen LS and Naveh-Floman N. Glaucoma in Fuchs's heterochromic cyclitis associated with congenital Horner's syndrome. Br J Ophthalmol 1987, 71: 844-9. 
${ }^{35}$ Alanko HI, Vuorre I, Saari KM. Characteristics of corneal endothelial cells in Fuchs's heterochromic cyclitis. Acta Ophthalmol 1986, 64: 623-31.

${ }^{36}$ Brown SI. Peripheral corneal edema after cataract extraction. Am J Ophthalmol 1970, 70: 326-8.

${ }^{37}$ Waardenburg PJ. Franceschetti A, Klein D. Genetics and Ophthalmology. Blackwell, Oxford 1961.

${ }^{38}$ Duke-Elder S. Pigmentation. In: System of Ophthalmology Vol III (Normal and Abnormal Development). Henry Kimpton, London 1966.

${ }^{39}$ Makley TA. Heterochromic cyclitis in identical twins. Am J Ophthalmol 1956, 41: 768-72.

${ }^{40}$ Saari M, Vuorre I, Tiilikainen A, Algvere P: Genetic background of Fuchs's heterochromic cyclitis. Can J Ophthalmol 1978, 13: 240-46.

${ }^{41}$ Posner A and Schlossmann A: Syndrome of unilateral attacks of glaucoma with cyclitic symptoms. Arch Ophthalmol 1948, 39: 517-33.
${ }^{42}$ Murray PI, Dinning WJ, Rahi AHS: Contrasting relations between suppressor cell number and function in acute anterior uveitis and heterochromic cyclitis. Acta Ophthalmol 1984, (Suppl) 163: 52.

${ }^{43}$ Kruit PJ: Cellular immunity against the $54 \mathrm{KD}$ corneal epithelium antigen in chronic ocular diseases. In: Corneal autoimmunity. A clinical and experimental approach. VU Uitgeverij, Amsterdam 1987.

${ }^{44}$ Gaag, R van der, Broersma L, Rothova A, Baarsma S, Kijlstra A: Immunity to a corneal antigen in Fuchs's heterochromic cyclitis patients. Invest Ophthalmol Vis Sci 1989, 30: 443-8.

${ }^{45}$ Murray PI, Hoekzema R, van Haren MAC, Luyendijk L, Kijlstra A: Aqueous humour analysis in Fuchs's heterochromic cyclitis. Current Eye Res 1990, 9: 53-7. 